
\title{
BResarch Soute \\ TACA: Trust Aware Clustering using ACO for Secure and Reliable Vehicular Ah hoc Network Routing
}

\section{Megha Vishal Kadam ( $\square$ megha.desai1@gmail.com )}

Shri Jagdishprasad Jhabarmal Tibrewala University https://orcid.org/0000-0002-3240-9033

\section{Vinod M Vaze}

Shri Jagdishprasad Jhabarmal Tibrewala University

\section{Satish R Todmal}

Shri Jagdishprasad Jhabarmal Tibrewala University

\section{Research Article}

Keywords: Ant colony optimization, clustering, cluster head, degree of connectivity, security, trust parameters, vehicular ad hoc network

Posted Date: July 29th, 2021

DOl: https://doi.org/10.21203/rs.3.rs-710906/v1

License: (9) This work is licensed under a Creative Commons Attribution 4.0 International License. Read Full License 


\title{
TACA: Trust Aware Clustering using ACO for Secure and Reliable Vehicular Ah hoc Network Routing
}

\author{
Megha V Kadam*, Research Scholar, Shri.JJT University, Churella, Jhun- \\ jhunu(Rajasthan), megha.desai1@gmail.com \\ Vinod M Vaze, Professor and Ph.D guide, Shri.JJT University, Churella, Jhun- \\ jhunu(Rajasthan), vinod.vaze@gmail.com \\ Satish R Todmal, Ph.D guide, Shri.JJT University, Churella, Jhunjhunu(Rajasthan), \\ srtodmal@gmail.com
}

\begin{abstract}
In the modern era, the Vehicular Ad-hoc Network (VANET) received significant attention for information sharing among the societies. The emerging Internet of Things (IoT) for smart city perspective boosts the development of VANET based applications such as road safety and Intelligent Transport System (ITS). The efficiency of such networks is a widely studied research problem. The clustering has shown an efficient technique to address the challenges of VANET QoS and computational efficiency. The vehicles are grouped according to certain conditions to form the cluster. In this way, the entire network divides into different clusters. Each cluster consists of limited vehicles with its leader called Cluster Head $(\mathrm{CH})$. But the major challenge for VANET clustering has related to the stability of the cluster. Due to high network dynamics, the unreliability for $\mathrm{CH}$ selection and data relaying becomes a security threat in VANET. To address such a security threat of VANET clustering, we proposed TrustAware Clustering using Ant Colony Optimization (TACA) protocol. For each cluster, an ACO-based optimal $\mathrm{CH}$ selection algorithm applying different trust components of the vehicle. The ACO solves the problem of optimal $\mathrm{CH}$ selection with minimum control overhead and maximum $\mathrm{CH}$ lifetime. The optimal $\mathrm{CH}$ selected has been selected based on trust-aware ACO fitness function using the parameters such as vehicle speed, Degree of Connectivity (DoC), vehicle congestion, and Packet Relaying Probability (PRP). This mechanism enables clusters to select reliable $\mathrm{CH}$ to address the security concerns of VANET communications. The TACA protocol has been evaluated with recent similar methods, and the results demonstrate efficiency in terms of QoS and computational overhead of clustering.
\end{abstract}

Keywords: Ant colony optimization, clustering, cluster head, degree of connectivity, security, trust parameters, vehicular ad hoc network.

\section{Introduction}

Vehicular Ad hoc Networks (VANETs) are encouraging that perform a vital part in Intelligent Transportation System (ITS). VANETs support vehicle operators to 
communicate (by allowing Vehicle-to-Vehicle (V2V) plus Vehicle-to-Infrastructure (V2I) connections) to withdraw several dangerous driving conditions. VANETs carry a variety of security credentials such as co-operative travel monitoring, administration of traffic streams, accidental intersection, restriction of collisions, nearby knowledge services, including real-time road routes calculation. VANETs have two things: vehicles described On-Board units (OBUs) and access points described Road Side Units (RSUs). RSUs are set and can act as a distribution point for vehicle networks [1].

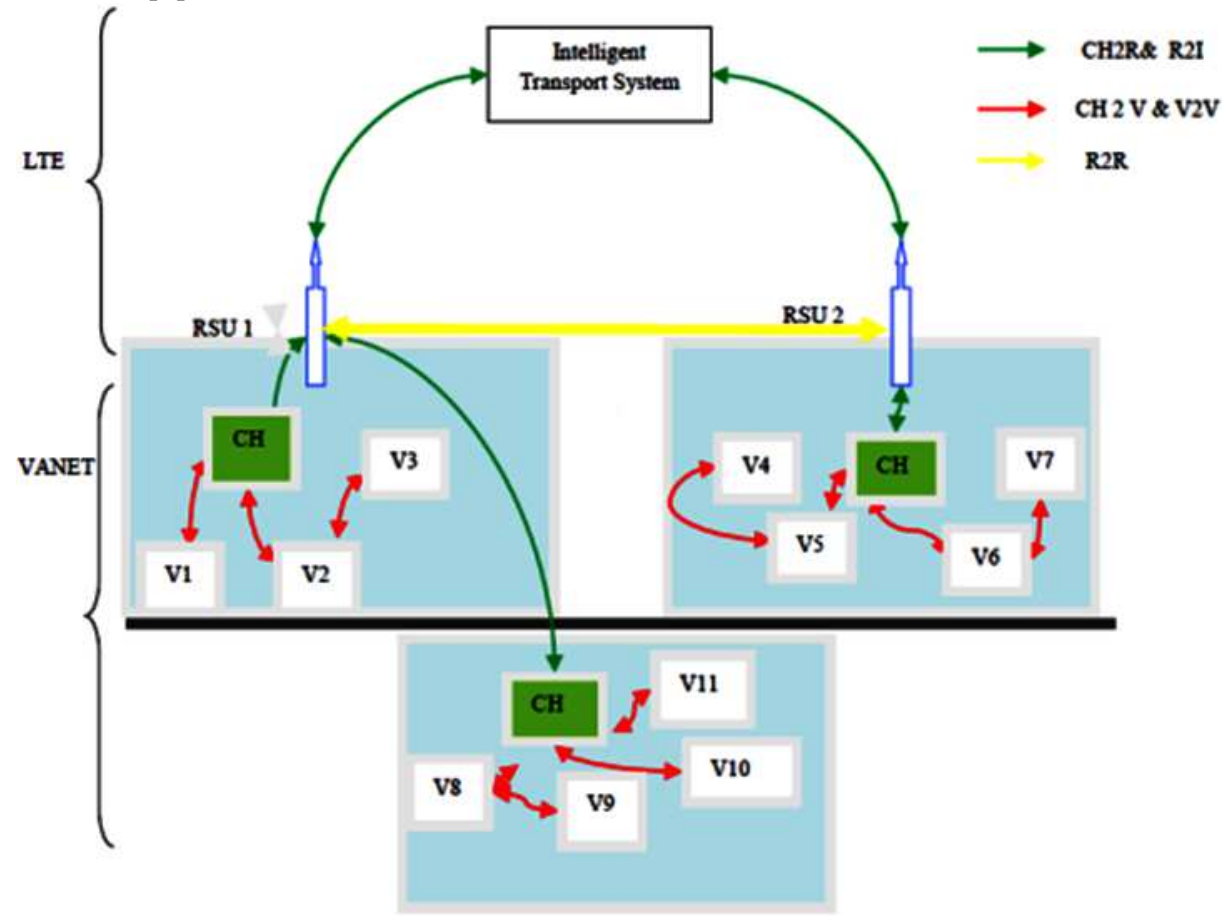

Figure 1. Example of VANET Topology and Communications

Figure 1 shows the important things of the VANET such as vehicles, RSUs, and OBUs, where they communicate data by using the V2V, V2I, and infrastructure-toinfrastructure (I2I) manner. Those connections have been supported by dedicated short-range communication (DSRC) [2]. The vehicular communication scheme has been accessed by the standard IEEE 802.11p that supports Wireless Access in Vehicular Environments (WAVE) [3]. The IEEE 802.11p establishes the link layer that encourages internet protocol and the WAVE Short Message Protocol (WSMP). The WAVE standard is utilized to reduce dangerous conditions such as restriction or identification of the appearance of accidents. The ITS utilizes the WAVE protocol to announce information such as climate circumstances, roadways support, and road traffic situations [4].

In the past decade, increased requirements of road safety and efficient road transportation system have encouraged vehicle companies to combine wireless interfaces and networking into transports. Along with road safety purposes, VANETs produce 
Internet connectivity to vehicles while on transit, so travelers can download a song, transmit emails, book restaurants, and/or perform games. Because of the vehicle's high mobility, VANETs are characterized by fast topology variations. The latter causes planning an effective routing protocol for the vehicular conditions extremely challenging. Due to the open nature of VANETs, they are vulnerable to various security threats as such networks are mainly dependent on control, communication, and computing technologies [3] [4]. The fault or malicious vehicles in VANET may lead to accidents and public assets lost due to miscommunications based on vehicle sensor data. Therefore, if the detection of such sensor data is not effectively handled, then it may lead to traffic jams, road accidents, etc as most vehicles are wrongly redirected by face traffic alerts. Thus providing reliable communications among the V2V or V2I is the first research problem. On the other hand, VANET is similar to MANET, but the routing protocols of MANET such as reactive, proactive, or hybrid cannot directly apply to VANET as high network dynamics due to the high mobility speed of vehicles. Frequent and high vehicle movement causes the communication connections among vehicles to be broken. These link failures raising the broadcasting and routing control overhead. It is commencing to degeneration of the protocol Quality of Service (QoS). The high dynamics of vehicles have not addressed MANET routing protocols [5].

Thus, designing adaptive routing protocols for VANET to handle such network dynamics effectively is a challenging research problem. Several attempts were made on applying the MANET protocols on VANET, but those studies have shown that Optimized Link State Routing (OLSR) has better performance than Ad-hoc Ondemand Distance Vector (AODV) and Destination Sequenced Distance Vector (DSDV), and Dynamic Source Routing (DSR). The performance investigations of conventional ad-hoc routing protocols like AODV, DSDV, and DSR for some highway situations have been analyzed in [2]. The authors showed that those routing protocols do not fit for VANETs. Performance of MANET protocols noted that traditional routing protocols possess costlier control overhead which causes reduced Packet Delivery Ratio (PDR). The clustering-based routing protocol solves the problems associated with the routing protocols such as AODV, DSR, etc. The cluster-based routing protocols are effective techniques for VANETs.

Several clustering mechanisms were designed earlier for networks like Wireless Sensor Networks (WSNs) and MANET [6] [7]. A similar approach with VANET specific parameters for cluster formation and Cluster Head $(\mathrm{CH})$ selection has been designed for VANET QoS enhancement [8-13]. The clustering demonstrates an imminent approach for form the group vehicles and organizes the wireless communications effectively. Clustering implies gathering vehicles dependent on some regular qualities, for example, topographical area, speed, the course of development, and so forth. These techniques make the VANETs more robust and scalable. Cluster-based approaches might be the practical answers for supporting adaptable multihop communication for high-density VANETs. Nonetheless, considering the highclass highlights of VANETs, customary clustering methods are wrong for VANETs. A few stable grouping-based methods in VANETs have been proposed for stable clustering. But the significant issue for designing clustering techniques is that to achieve the long life of clusters and reliable $\mathrm{CH}$ selection, and reliable data 
transmission for unreliable network VANET conditions higher mobility of vehicles, congestions, and longer geographical distances. Thus designing the reliable clustering protocol to ensure the QoS improvement for unreliable VANET conditions with minimum computational burden and control overhead is still a research challenge.

In this paper, a Trust Aware Clustering using ACO (TACA) routing protocol has been proposed to overcome the challenges of VANET communications. Trustbased clustering is distributed mechanism that enables evaluation of each vehicle using its trust parameters to select reliable $\mathrm{CH}$ for authentic and secure clustering in VANET [14] [15]. The trust-based approach supports the capability of distinguishing unreliable and reliable vehicles. The core focus of the TACA protocol is to solve the problem of reliable $\mathrm{CH}$ selection for each cluster in VANET. To address this problem, we designed the nature-inspired algorithm Ant Colony Optimization (ACO) with a modified fitness function. Compared to other optimization techniques, ACO is a metaheuristic technique that provides solutions with minimum overhead and simple to solve the clustering problem for VANET. The fitness function of ACO has been designed to select the most reliable and optimal vehicle as $\mathrm{CH}$ for the current cluster using the direct and indirect trust parameters of each vehicle. After cluster formation, the periodically computed trust-based fitness values of each vehicle have been utilized for reliable inter-cluster and intra-cluster data transmission. Section 2 focused on a brief review of various clustering techniques of VANET with the motivation and contributions of this paper. Section 3 presents the design of the proposed protocol. Section 4 presents an investigation of simulation results. Section 5 presents the conclusion and suggestions for future work.

\section{Related Works}

This section presents a brief study of various VANET clustering mechanisms proposed during the last decade. First, we reviewed the existing clustering techniques progressively according to their appearances and then discussed the research gaps followed by the proposed contributions of this paper.

\section{A. State-of-Art Methods}

The first clustering for VANET of choosing group setup parameters by utilizing the physical situation of the vehicles proposed in [16]. A gathering of vehicles picks a $\mathrm{CH}$, but the span of the group is controlled by the $\mathrm{CH}$. The multi-hop clustering methodology proposed in [17] with another improvement metric requiring the similar versatility between vehicles in a multi-bounce isolated, picking the vehicle with the base aggregate portability esteem as the group head. In [18], Hierarchical Clustering Algorithm (HCA) was proposed for the development of clusters with a scope of the most extreme four bounces. This protocol schedule transmissions and channel access inside the cluster to guarantee dependable correspondence. The SBCA protocol [19] makes clusters with a progressively steady structure by considering the portability, the number of neighbors, and the initial term of the vehicle. The Re- 
gion-based Clustering Mechanism (RCM) is introduced in [20] to enhance the scalability of MAC protocol for VANETs. In RCM, the network is apportioned into various space division units, and every division unit is restricted to a fixed number of vehicles for keeping away from disputes of channels. The multi-administratordriven great clustering system was proposed in [21]. It showed a dynamic bunch creation system that contains overwhelming weight settled and light-weight portable administrators and groups the vehicles that show related versatility models, headings, and speeds. Another novel clustering-based proposed for VANET in [22]. They designed a clustering calculation that considers both center point position and center immovability in VANETs. The strategy hopes to make stable clusters by diminishing re-clustering overhead, hauling out cluster lifetime, and shortening the ordinary division between $\mathrm{CHs}$ and their CMs. Mainly fundamental, this calculation response to single and distinctive $\mathrm{CHs}$. The optimization (ACO) based clustering protocol for VANET proposed in [23] called Clustering calculation Ant Colony Optimization (ACO) for VANETs (CACONET). CACONET shapes enhanced clusters for lively discourse. In [24], the FAST clustering protocol proposed to outline established clusters for deferment constrained applications. The system intends to restrain the $\mathrm{CH}$ race and the cluster part decision latencies. The static geographical clustering algorithm proposed in [25] outlines static geological clusters as demonstrated by the width of the city which ends up being more gainful and constant $\mathrm{CH}$ mastermind over the larger piece of its execution estimations among various calculations. The fuzzy logic-based $\mathrm{CH}$ selection and cluster formation algorithm was proposed in [26] using key parameters such as speed, distance, acceleration, and direction. Another novel clustering method was proposed for the VANET environment in [27] with the point of improving the stability of the system cluster using the mobility speed mainly. In [28], a new dynamic mobility-based and stability-based clustering plan was presented for an urban city situation. The proposed conspire applies the vehicle's moving direction, relative position, and connection lifetime estimation. The novel algorithm proposed in [29] for the way toward addressing the challenges of cluster formation and $\mathrm{CH}$ selection appropriate for VANETs. Three different algorithms such as cluster-based life-time routing (CBLTR) protocol, Intersection dynamic VANET routing (IDVR) protocol, and control overhead reduction algorithm (CORA) proposed in [30] for VANETs. The $\mathrm{CH}$ selection was performed by maximum lifetime parameter among all the vehicles. The Density-Based Dynamic Clustering (DBDC) protocol was introduced recently in [31]. They designed a strategy to discover the accurate location in the lane for congestion management. They used the threshold of average vehicle density of each cluster for congestion control. The trust-based clustering protocol called Double Head Clustering (DHC) had proposed in [32] for VANET. They computed the trust parameters for optimal $\mathrm{CH}$ selection such as vehicle mobility, direction, and position along with signal-to-noise ratio (SNR) and link expiration time (LET). Recently, the author addressed the challenges of stable and reliable clustering for VANET in [33]. They proposed a StabTrust protocol using different trust parameters. The trustbased mechanism has been designed for selecting the reliable $\mathrm{CH}$ in each cluster. Their trust mechanism comprises the experience, reputation, and knowledge of the node. The optimization technique recently applied for VANET clustering in [34] is 
called Grasshoppers Optimization Algorithm (GOA). The GOA has designed for optimal $\mathrm{CH}$ selection using inter-vehicle distance and route length parameters for fitness computation. However, GOA failed to consider the reliability of VANET communications in terms of cluster stability by considering the mobility and congestion security threats.

\section{B. Motivation}

Notwithstanding the inherent benefits of recent VANET clustering protocols, there are nevertheless various current challenges for VANET clustering. The research gaps noticed from the above literature are:

- Efficient techniques have been required for VANET communications with the minimum computational burden to support network scalability and prevent data loss [35] [36].

- The multi-level clustering methods have not yet exploited a completely exploited and challenging approach as it depends on different parameters to build a cluster [31] [37].

- The trust-based clustering confirmed effective solutions for VANET clustering and routing, but lack of optimization in vehicle selection leads to higher control overhead and data loss problems [32] [33] [38].

- The unreliable and unauthenticated RSUs dependent trust-based clustering techniques also lead to significant control overhead and security challenges [33].

These research gaps motivate us to propose the TACA protocol in this paper. The possible contributions of the TACA protocol are:

- We formulate the problem of selecting the reliable $\mathrm{CH}$ node for each clustering using the ACO algorithm to maximize the life of the cluster with minimum computational burden and communication delay.

- The trust-based fitness function has been used in ACO to select the reliable vehicle as $\mathrm{CH}$ of each cluster. To achieve scalability and reliability requirements, we computed both direct and indirect trust parameters for computing the integrated trust-based fitness value of each vehicle.

- The fitness score of each vehicle has been updated in its routing table entries periodically. These values have directly been accessed during intercluster and intra-cluster routing with aim of reducing the overhead and delay.

- To claim the scalability and reliability of the TACA protocol, we designed network scenarios with density and mobility variations using different mobility models and compared its performance with recent protocols. 


\section{$3 \quad$ Proposed Methodology}

As per the contributions mentioned in the above section, this section presents the functionality and design of the TACA protocol for VANET clustering. Figure 2 shows the working of the TACA protocol that comprises cluster formation, optimal $\mathrm{CH}$ selection, and reliable data transmission phases. After network deployment, the VANET partitioned into different clusters periodically using the conventional $\mathrm{K}$ means clustering. We used this approach over the other dynamic techniques of the number of clusters formation because of its simplified and lightweight mechanism. Discovering the optimal number of clusters is out of the scope as the main problem is concerning reliable and stable $\mathrm{CH}$ selection for each cluster. After network portioning, an ACO-based $\mathrm{CH}$ selection algorithm has been processed for each cluster. The ACO has applied to select the reliable $\mathrm{CH}$ using trust-based fitness function evaluation. The number of ants represents the number of vehicles that are part of the current cluster. The pheromone value (fitness value) for each ant (vehicle) has been updated in its routing table. The next phase belongs to reliable next-hop selection for intra-cluster and inter-cluster data transmission to secure the network data and prevent data loss. It can be performed by directly analyzing the periodic trust value of each vehicle. In subsequent sections, we present the complete design of the proposed model. The process of re-clustering and cluster maintenance will perform periodically according to Time Division Multiple Access (TDMA) channel schedules to maintain network reliability, improve cluster lifetime, and guaranteed QoS.

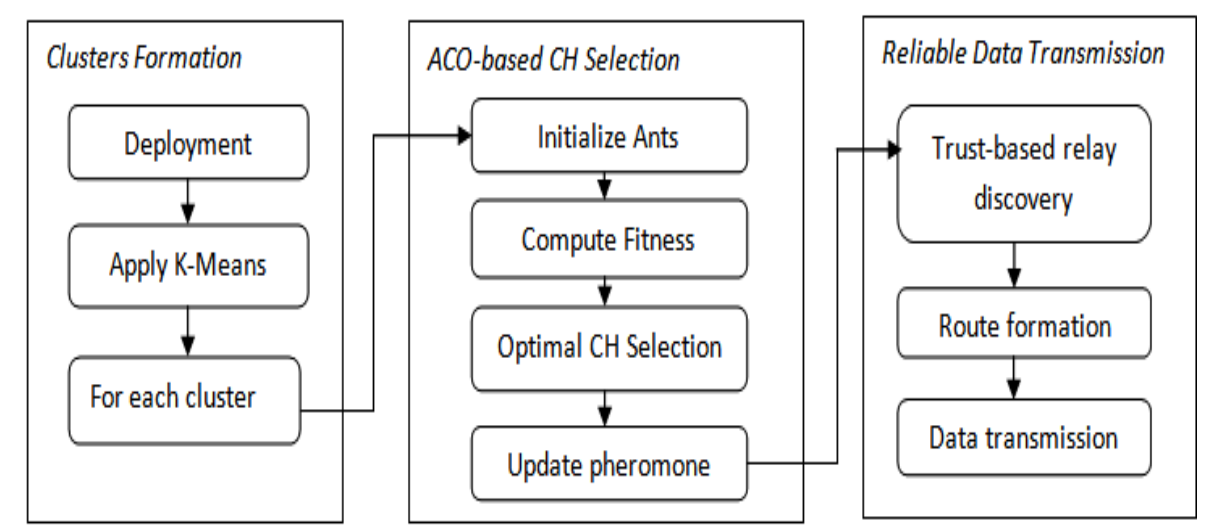

Figure 2. Functionality of proposed TACA protocol

\section{A. System Design and Assumptions}

We define the system model for TACA protocol as: Let $N$ number of vehicles with $M$ number of RSUs deployed in network of $X \times Y$ size. After deployment, the initial $C$ number groups formed by using K-means clustering algorithm i.e. $\left\{c^{1}, c^{2}, \ldots, c^{C}\right\}$. For each $q^{t h}, q \in C$ cluster, optimal and reliable $\mathrm{CH}$ selection algorithm applied using ACO to maximize the network QoS and cluster stability in presence of the number of unreliable threats in the network. Before presenting the 
ACO-based $\mathrm{CH}$ selection algorithm, the design of the TACA protocol is based on some assumptions such as:

- The VANET consists of $N$ number of vehicle nodes deployed randomly with capability of moving around the mobility pattern.

- $\quad$ The VANET consists of $M$ number of RSUs situated at fixed positions in network without any movement.

- All vehicles are homogenous, mobile and having a unique ID without energy constraints.

- $\quad$ The k-means clustering applied to partition the network for each clustering round.

- The data transmissions performed V2V manner in that Cluster Members (CMs) transmit their data to their corresponding $\mathrm{CH}$ nodes.

- $\quad$ The $\mathrm{CH}$ nodes receive periodic data from its $\mathrm{CMs}$ and aggregate data by using efficient data aggregation strategy.

- The data transmissions performed V2I manner in that CHs transmit their data to their corresponding RSU nodes.

- $\quad$ The multi-hop and symmetric manner communications applying among the V2V and V2I links.

- The geographic position and distance parameters estimated by using the Global Positioning System (GPS) for each vehicle.

\section{B. Reliable CH Selection}

As per the model designed with assumptions, the core contribution of the TACA protocol has trust-based reliable $\mathrm{CH}$ selection using the robust $\mathrm{ACO}$ technique. The process of secure and optimal $\mathrm{CH}$ selection using the trust computation proposes to overcome the challenges of previous methods. To prevent data loss and network unreliability conditions, it is vital to select the vehicle as $\mathrm{CH}$ that is more trustworthy among other vehicles. As the trust score should consider both the security and clustering QoS perspectives, we used direct (packet forwarding ratio) and indirect trust (mobility, degree of connectivity, and vehicle congestion) parameters. To solve the problem of optimal, stable, and reliable $\mathrm{CH}$ selection, we applied the ACO algorithm using a modified fitness function. The fitness of each ant of the ACO algorithm has computed using direct and indirect trust parameters. Due to the significant benefits of using the ACO in recent studies across various routing and cluster challenges of different networks, we exploit the use of ACO to select the optimal $\mathrm{CH}$ with minimum computational efforts compared to other optimization techniques. The function of ACO is the modeling of the ant behavior related to their ability to find the best available vehicle from the anthill to the food source (reliable and stable $\mathrm{CH}$ selection) as demonstrated in figure 2 (1) where the problem definition is represented. The process of ACO is iterative in which initially the ant broadcasted the way with pheromone, and this information utilized by other ants to select the optimal CH (figure 2 (2)). The final solution by the ant is then visible when all the possible options are evaluated (figure 2 (3)). Thus the steps of ACO for optimal CH selection are summarized as: 


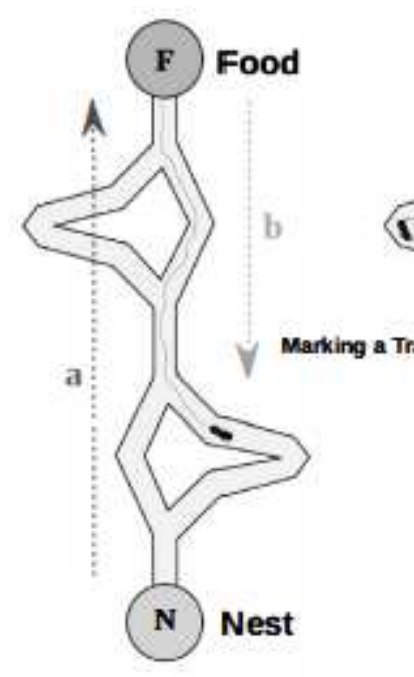

1

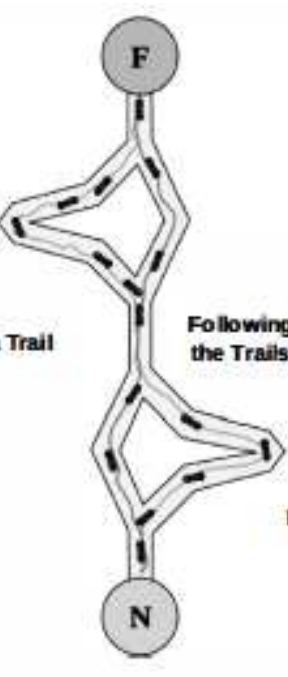

2

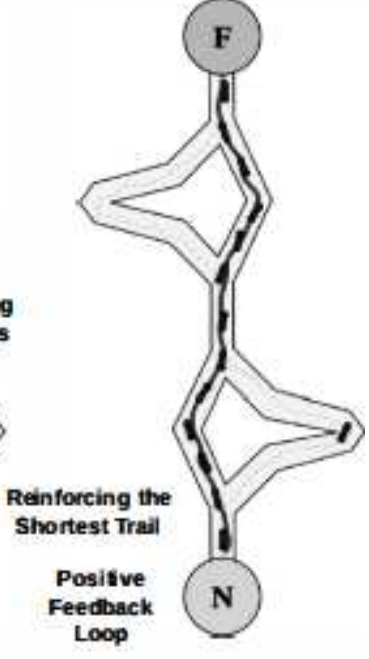

3

Figure 2. Functionality of ACO algorithm

- For each cluster, initialize the number of ants and other parameters related to iteration and temporary best solution.

- Construction of the acceptable alternative $\mathrm{CH}$ solutions.

- Compute the fitness of each Ant until the convergence criteria are satisfied and update the current solution of $\mathrm{CH}$ selection.

- Update the pheromone value in the routing table related to each ant.

- Return the optimal CH selection after convergence criteria are achieved.

Figure 3 and algorithm 1 represent the flowchart and pseudo code of the proposed ACO-based $\mathrm{CH}$ selection approach of the TACA protocol respectively. For each cluster, ACO initialize the parameters such as number of iterations Ite and $k$ number of ants $\left\{a^{1}, a^{2}, \ldots a^{k}\right\}$ for $n$ number of vehicles of the current cluster. The group of $k$ number of ants deployed to search the solution of a problem. At each iteration Ite, every ant $a^{i}, i \in k$ builds its own problem-solution based on the rules. In TACA, the solution is the selection of reliable $\mathrm{CH}$ among all available options based on the trust-based fitness function to establish a stable clustering solution for VANET. At every iteration Ite, ACO performs the three steps to select stable and reliable $\mathrm{CH}$ for each cluster such as (1) finding solution by each ant, (2) pheromone is deposited, and (3) pheromone evaporation occurs. 


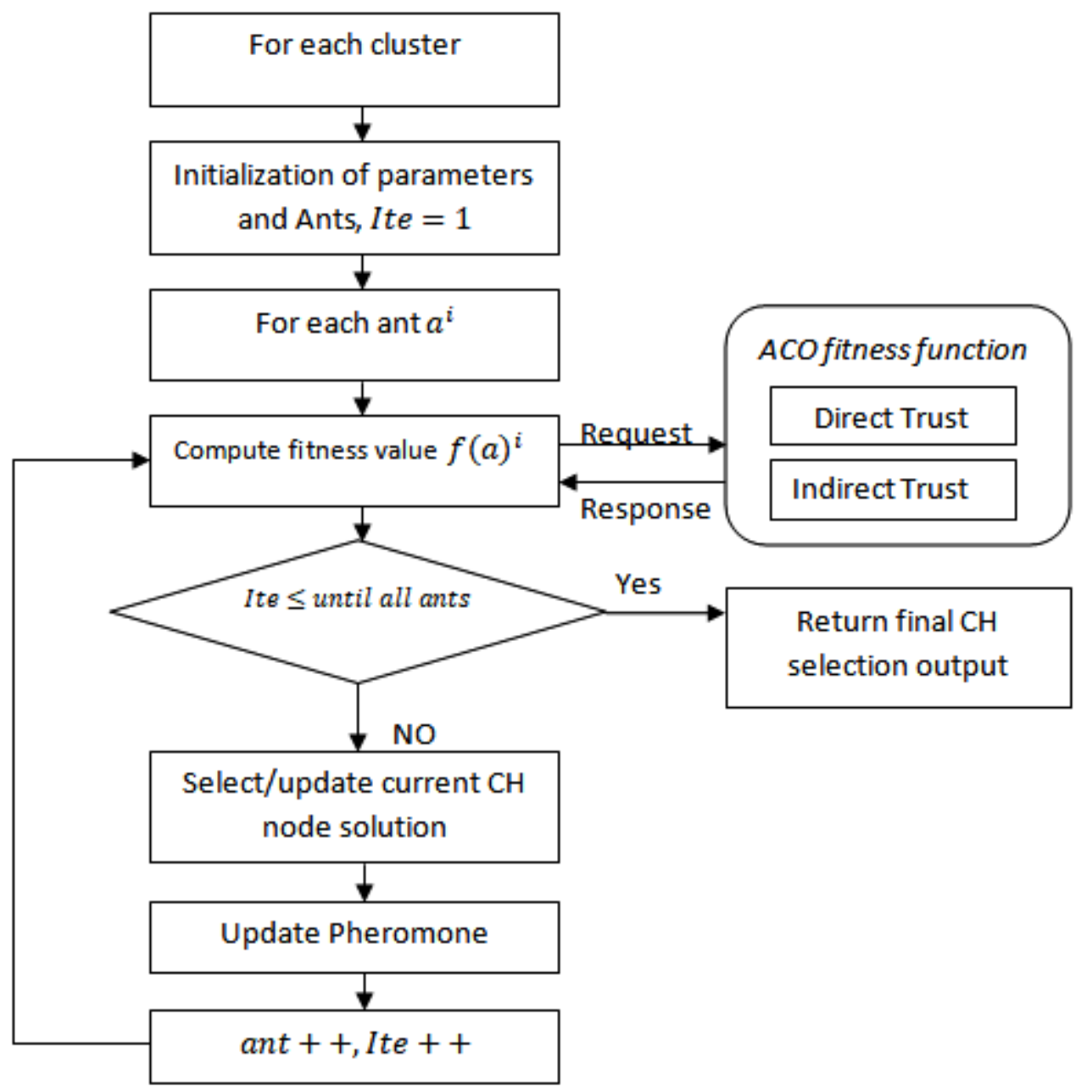

Figure 3. ACO-based reliable and stable $\mathrm{CH}$ selection

\begin{tabular}{|l|}
\hline Algorithm 1: Reliable CH selection using ACO \\
\hline Inputs \\
M: Number of clusters \\
CM: set of nodes in each cluster \\
RT: Routing table entry for each vehicle \\
Output \\
CH: set of cluster heads selected during current round \\
\hline 1. For each TDMA schedule \\
2. For each cluster $j=1: M$ \\
3. Initialize parameters: \\
4. $\quad C H^{J}=N U L L$ \\
5. $\quad k=\operatorname{size}(C M(j))$ \\
\hline
\end{tabular}




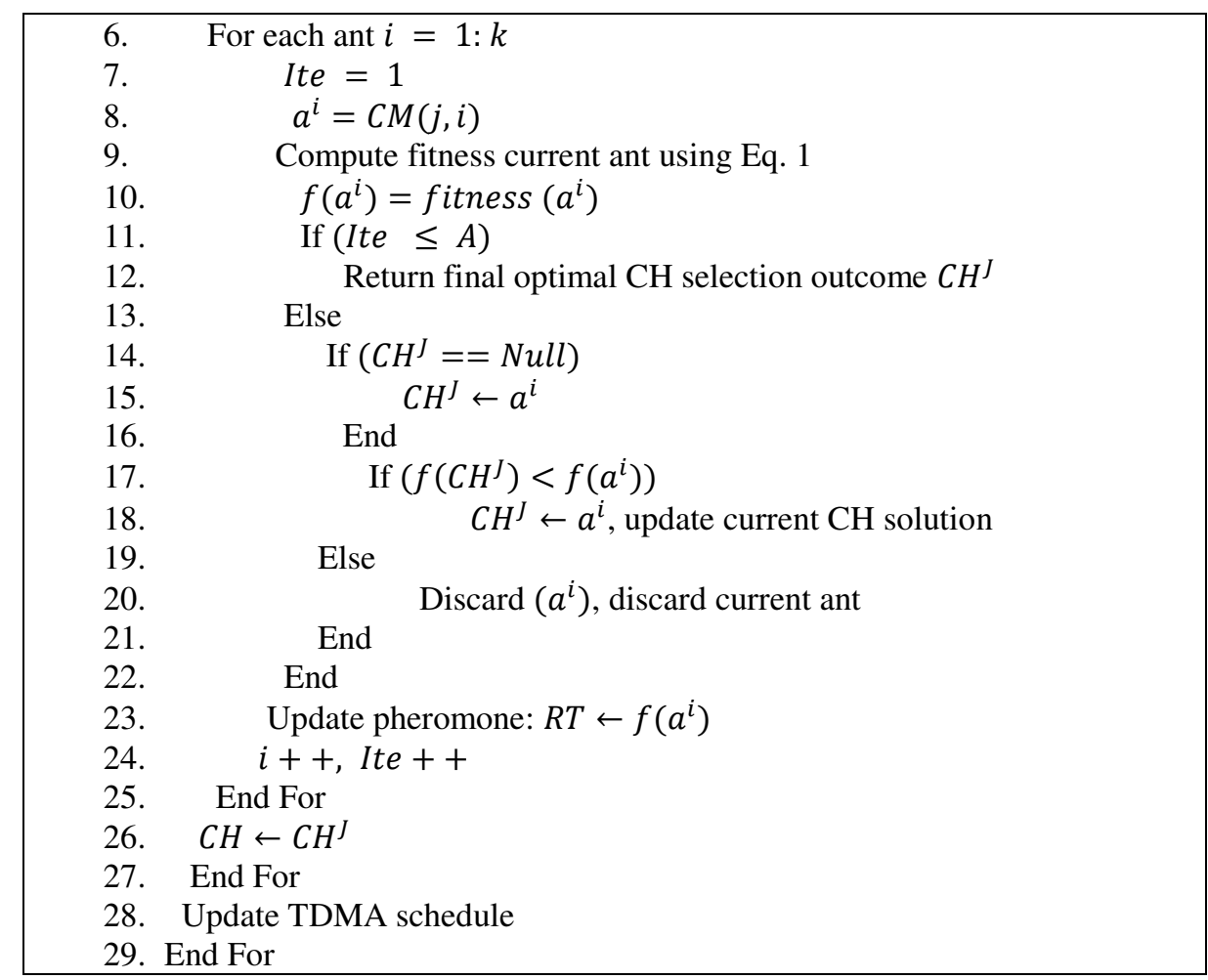

As showing in algorithm 1 and figure 3, the periodic process of electing the optimal $\mathrm{CH}$ selection has been performed by evaluating each ant belonging to each cluster using trust-based fitness value. The core part of this algorithm is computation of fitness value of each ant using the direct and indirect trust value. The fitness value $f\left(a^{i}\right)$ of $i^{\text {th }}$ ant belonging to $j^{\text {th }}$ cluster has computed by:

$f\left(a^{i}\right)=\left(w^{1} \times D\left(a^{i}\right)\right)+\left(w^{2} \times I D 1\left(a^{i}\right)\right)+\left(w^{3} \times I D 2\left(a^{i}\right)\right)+\left(w^{4} \times I D 3\left(a^{i}\right)\right)$

(1)

Where, $D\left(a^{i}\right)$ represents the direct trusts score for packet forwarding ratio parameter. ID1 $\left(a^{i}\right), I D 2\left(a^{i}\right)$, and $I D 3\left(a^{i}\right)$ represents indirect trust scores for three parameters mobility speed, degree of connectivity, and congestion respectively. The $w^{1}-$ $w^{4}$ represents the normalization factors to scale the values of each trust score in between 0 to 1 so that final value of $f\left(a^{i}\right)$ within range of 0 to 1 .

Direct Trust Computation: The packet forwarding ratio allows estimating the legitimate nodes accurately by computing direct trust value. Node $a^{i}$ forwards data packets of from another vehicle $a^{j}$ and broadcasts 4 ACK packets. During this time, node $a^{j}$ collects such ACK packets of node $a^{i}$ to estimate the number of sensing packets. The rate of data transmission packets computed at time $t$ as:

$f\left(a^{i}\right)=\frac{S P\left(a^{i, j}(t)\right)-S P\left(a^{i, j}(t-1)\right)}{S P\left(a^{i, j}(t)\right)+S P\left(a^{i, j}(t-1)\right)}$ 
Where, $S P\left(a^{i, j}(t)\right)$ is number of successful packets forwarded by $a^{i}$ at time $t$. This parameter efficiently secures the network from unreliable vehicles in network as well as estimate the malicious behaviour of node. Higher $f\left(a^{i}\right)$ value results into higher chances of $a^{i}$ as reliable vehicle for $\mathrm{CH}$ selection.

Indirect Trust Computation: Three indirect trust parameters compared for each ant corresponding to a particular cluster.

- Mobility: It is a vital parameter for VANET clustering and routing, thus selecting the vehicle with less moving speed will be the reliable solution and it prevents data loss. The first parameter is the mobility of the vehicle that computed at current $t$ as:

ID $1\left(a^{i}\right)=$ mobility $\left(a^{i}, t\right)$

Where, the mobility function estimates the mobility speed of $a^{i}$ at time $t$. The speed function is based on speed prediction technique using previous moving speed history of vehicles. The locations of vehicle retrieved using the GPS technology. The $I D 1\left(a^{i}\right)$ represents the node speed of $i^{\text {th }}$ vehicle for $j^{\text {th }}$ cluster, and it is normalized as:

$I D 1\left(a^{i}\right)=\frac{\left(\lambda-I D 1\left(a^{i}\right)\right)}{\lambda}$

Where, $\lambda$ is represents the maximum mobility value. In our simulation we kept the maximum pre-defined value as $140 \mathrm{~km} / \mathrm{h}$. Higher the value of $I D 1\left(a^{i}\right)$, less mobility speed of vehicle $a^{i}$, thus, higher chances to become $\mathrm{CH}$.

- Connectivity: This parameter ensures the reliability of clustering and data transmission phase as it helps to select the node with number of neighbourhood nodes. The number of neighbourhood nodes of $a^{i}$ defines its connectivity that is computed as at time $t$ :

Connectivity $\left(a^{i}(t)\right)=\left[\frac{n_{i}}{\operatorname{dist}\left(a^{i}, a^{j}\right)}<\right.$ rssi $]$

Where, $i \neq j$, dist $\left(a^{i}, a^{j}\right)$ represents geographical distance between two nodes and Received Signal Strength Indicator (rssi) represents the communication range of node $i$. $n_{i}$ represent the count of connectivity. The connectivity parameter further normalized as:

ID $2\left(a^{i}\right)=1-\left(\frac{1}{\text { Connectivity }\left(a^{i}(t)\right)}\right)$

This parameter helps to select optimal $\mathrm{CH}$ as it takes minimum time for $\mathrm{CM}$ to $\mathrm{CH}$ data transmission. It also limits the malicious activities during periodic data transmissions within cluster, therefore, it is selected as trust parameter in TACA protocol.

- C. Congestion: For VANET, congestion or data traffic is another challenge for intelligent clustering protocol. The node with higher congestion is considered unreliable to become $\mathrm{CH}$. The unreliable node is frequently broadcasting routing packets that lead to traffic around it. It can also result in data loss and high control overhead in VANET communications. Therefore, we selected congestion estimation of each vehicle for reliable $\mathrm{CH}$ selection. The congestion rate of vehicle $a^{i}$ is computed as: 
ID3 $\left(a^{i}\right)=1-\left(\frac{B_{\text {consumed }}\left(a^{i}(t)\right)}{B_{\text {assigned }}}\right)$

Where, $B_{\text {consumed }}\left(a^{i}(t)\right)$ represents the current number of packets processing at node $a^{i}$ at time $t$ and $B_{\text {assigned }}$ represents maximum bandwidth capacity of vehicle $a^{i}$ allocated. Higher the value of $I D 3\left(a^{i}\right)$, maximum chances of $a^{i}$ to become $\mathrm{CH}$.

\section{Cluster Maintenance}

It is the continuous process in which the clusters are checked for optimization. The cluster maintenance phase has initiated in the network in case of $\mathrm{CH}$ failure, joining of a new vehicle in the cluster of CM fails to exit. As the vehicle frequently moving in VANET, the CMs can travel from one cluster to another cluster. Thus, rather than performing the re-clustering for each TDMA cycle, we applied optimal $\mathrm{CH}$ selection for each cluster for each TDMA cycle. It ensures network connectivity and reliability regardless of cluster size dynamics. It also minimizes the control overhead and communication delay. Apart from this condition, at each periodical interval the status of selected $\mathrm{CH}$ checked to perform the re-election of $\mathrm{CH}$ based on one of the below conditions:

- If the current $\mathrm{CH}$ failed, then re-election of $\mathrm{CH}$ initiated.

- If the current $\mathrm{CH}$ fitness value has been suppressed by any other CM fitness value in the same cluster, then the current $\mathrm{CH}$ relinquish its role and become the $\mathrm{CM}$, and then $\mathrm{CM}$ with the highest fitness value becomes the new $\mathrm{CH}$ using ACO.

- If the current $\mathrm{CH}$ crosses its cluster width and enters into another cluster width, then re-election of $\mathrm{CH}$ initiated.

\section{Data Transmission}

This is a vital phase of VANET in which the periodic network information is collected and transmitted to corresponding destinations in a multi-hop manner periodically. To prevent data loss or congestion, we used the modified Shortest Path Tree (SPT) approach that considers short hop count to discover the route from the intended source and destinations. The modified SPT selects the forwarder or relay node according to its current fitness value. The node with a higher fitness value has elected for data relaying operation towards the destination. Once all the routes are discovered, the route with a minimum hop count is selected to initiate data transmission. The routing process has been regarded as two types of communication (1) from $\mathrm{CH}$ to the RSU via the other intermediate $\mathrm{CH}$ nodes, (2) from CMs to their $\mathrm{CH}$ node. The relay selection according to its trust factor ensures the reliability and data protection for VANETs.

\section{Simulation Results}

This section presents the simulation results and comparative analysis of the proposed TACA protocol. The TACA protocol along with the existing protocols was 
implemented in NS2 using different network scenarios. To claim the efficiency of the TACA protocol, we have selected three recent trust-based clustering protocols of VANET as CACONET [23], DHC [32], and StabTrust [33]. The CACONET protocol was selected because it is the first clustering protocol for VANET that used ACO for optimal $\mathrm{CH}$ selection using speed and direction parameters. The DHC protocol was designed using different trust parameters for $\mathrm{CH}$ selection without using an optimization technique. The StabTrust protocol was similar to DHC except for the trust computation of each vehicle performed by the RSU node for $\mathrm{CH}$ selection and also includes the cryptography operations for data transmission. While the implementation of StabTrust, we consider only the approach of RSUbased $\mathrm{CH}$ selection and clustering. The performances have been measured in terms of different performance metrics such as average throughput, PDR communication delay, and control overhead. The evaluations performed using two main network scenarios as (1) density variations using the Random Walk mobility model, (2) mobility variations using the Manhattan grid mobility model. Table 1 and 2 disclosed the details for both these scenarios.

\section{A. Vehicle Density Analysis}

The clustering protocol for VANET should be scalable with an increased number of vehicles. To analyze the impact of increasing density, we have designed four networks of a varying number of vehicles in the network such as 45, 95, 145, and 150 with 5 RSUs in each. Table 1 is showing the other simulation parameters of density variations. These networks with 5 RSUs deployed in the network. Figure 4-7 demonstrates the outcome of average throughput, PDR, communication delay, and control overhead using each VANET clustering protocol.

Table 1. Simulation parameters for density scenario

\begin{tabular}{|l|l|}
\hline Vehicle Density & $45,95,145$, and 195 \\
\hline Vehicle Velocity & $35 \mathrm{~km} / \mathrm{hr}$ \\
\hline Simulation Duration & 500 seconds \\
\hline Clustering Protocols & StabTrust, DHC, CACONET, and TACA \\
\hline MAC & $802.11 \mathrm{p}$ \\
\hline Propagation Model & Two-Ray Ground \\
\hline Area & 7050 x 7050 \\
\hline Mobility Model & Random Walk mobility model \\
\hline Traffic Pattern & Constant Bit Ratio (CBR) \\
\hline Communication Pairs & 6 \\
\hline
\end{tabular}




\begin{tabular}{|l|l|}
\hline Number of RSUs & 5 \\
\hline
\end{tabular}

Figure 4 demonstrates the average throughput analysis for four clustering protocols using CBR data transmissions. Average throughput calculates the mean of the total number of packets delivered per second. We first observed that as the vehicles increase, the throughput performance decreases. It is mainly because of increased clustering and routing operations with an increased number of vehicles for each protocol. The increased number of vehicles also affects the other parameters like reduced PDR (figure 5), increased communication delay (figure 6), and increased control overhead (figure 7). Among all four clustering protocols, the TACA protocol delivered the best throughput performance for each network compared to other protocols DHC, stabtrust, and CACONET. This is due to the simplified approach of cluster formation, ACO-based reliable $\mathrm{CH}$ selection, robust cluster maintenance, and reliable data transmission functions of the TACA protocol. The stable clustering and route formation in TACA compare to other protocols leads to the increased number of successful packet transmissions. The recent stabtrust delivered the worst throughput performance compared to other protocols as it heavily relied on RSU nodes for trust computation of vehicles in $\mathrm{CH}$ selection protocol. The ACO-based clustering in CACONET achieved stable clusters in VANET compared to the DHC method, thus it is having higher throughput compared to DHC and stabtrust. Figure 5 demonstrates the outcome of PDR performances with a similar trend that was noticed for average throughput. The trust-based mechanism used in optimal $\mathrm{CH}$ selection using ACO delivered more stable clustering results into reduced data loss in the TACA protocol. The similar trust scores were further utilized without any extra efforts during inter and intra-clustering data transmission in TACA. It is observed that TACA reduced the data losses for all VANET networks approximately by $7 \%$ compared to existing clustering protocols.

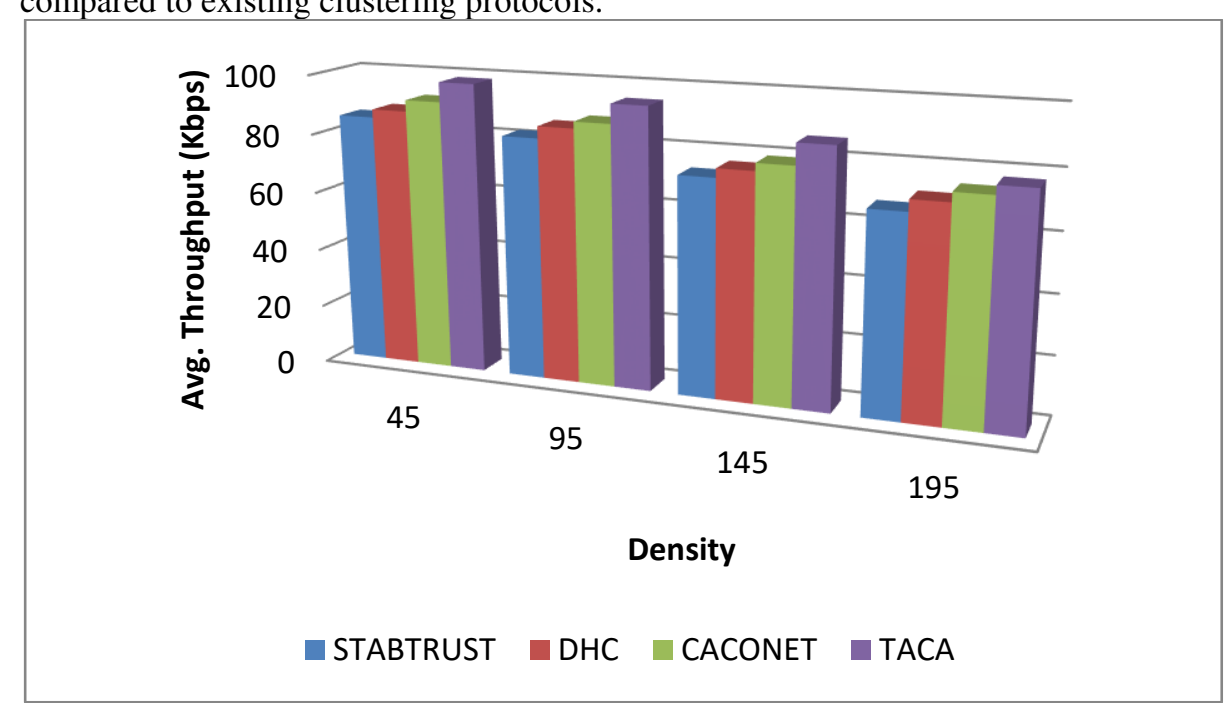

Figure 4. Average throughput analysis for density variations 


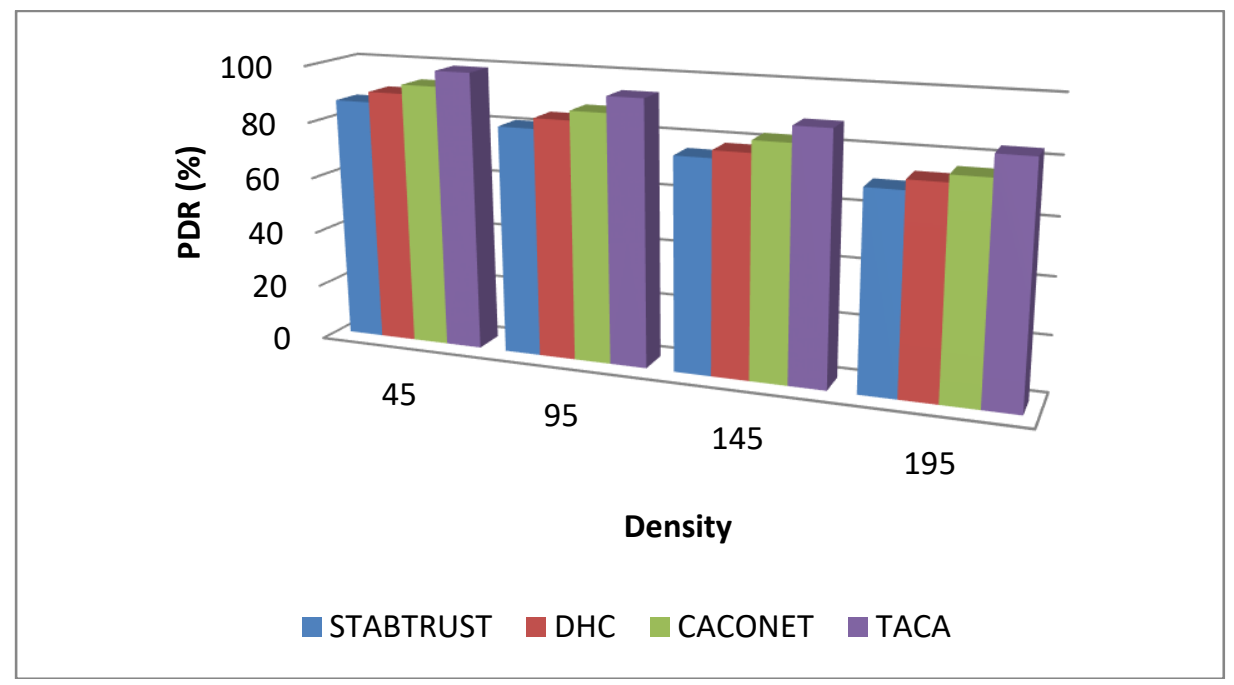

Figure 5. PDR analysis for density variations

Communication delay (figure 6) is another vital QoS parameter for VANET clustering and routing. These measurements compute the average time among the packet start time at all sources and the packet arriving at the time at all goal nodes. The reliable clustering with reliable data transmission in TACA leads to the minimum communication delay compared to all other existing clustering protocols. The communication delay mainly introduced due to frequent tasks of clustering and route formation in VANET, thus, TACA focused on ACO-based reliable and stable $\mathrm{CH}$ selection for clustering and trust-based reliable data relay section for routing in a simplified manner. This result in a significant reduction in delay performance of TACA compared to other protocols.

The existing methods DHC, CACONET, and StabTrust have limitations related to cluster formation and data routing. Non-optimized $\mathrm{CH}$ selection in DHC without reliable data transmission leads to unstable routes and clustering. CACONET used ACO only for clusters formation and optimal $\mathrm{CH}$ selection using only two trust parameters such as direction and velocity. StabTrust heavily relied on RSUs for accessing the trust parameters of each vehicle ad compute the integrated trust score periodically. This increases the high overhead of trust computation on the clustering process. In all existing protocols, congestion or packet forwarding ability behavior of vehicles cannot be considered for optimal $\mathrm{CH}$ selection. And hence, all protocols suffered from higher data loss, communication delay, and control overhead (figure 7) compared to the TACA protocol. Figure 7 demonstrates the control overhead performances of all protocols with density variations. Due to the ability of the TACA protocol to select the vehicle based on its velocity, congestion, connectivity, and transmission probability, control overhead minimized significantly. 

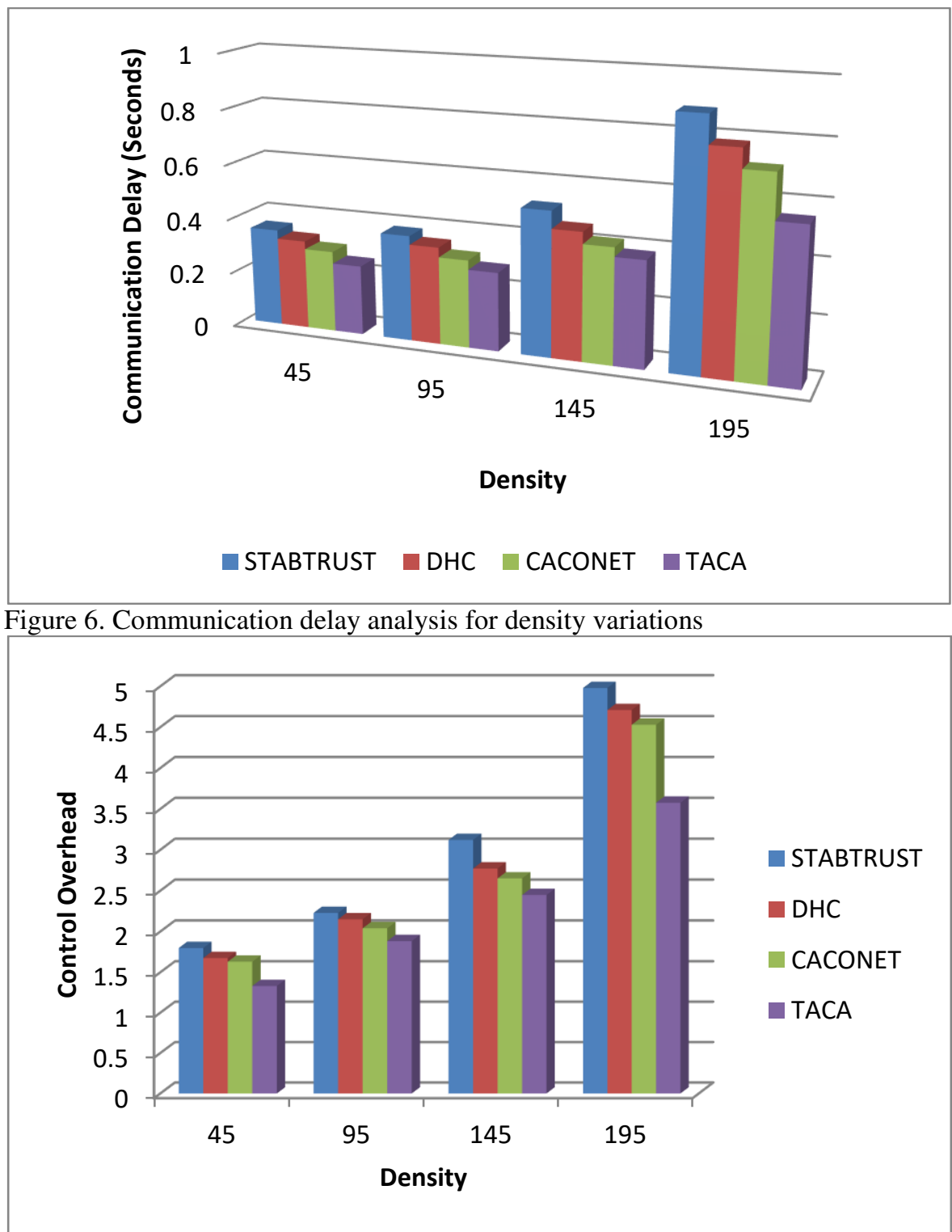

Figure 7. Control overhead analysis for density variations

\section{B. Vehicle Mobility Analysis}

The reliability and stability of VANET are significantly affected by vehicle mobility. The higher mobility leads to more unstable reliable network conditions due to frequent network dynamics. To conquer the challenges of higher mobility in VANET, we introduced the TACA protocol in this paper. We designed the networks of 45 vehicles and 5 RSUs with varying mobility speeds such as 15, 30, 45, 
60,75 , and $90 \mathrm{Km} / \mathrm{hr}$. Table 2 demonstrates the details of complete simulation parameters. Figures 8-11 are showing the impact of increased mobility on average throughput, PDR, communication delay, and control overhead respectively.

Table 2. Simulation parameters for mobility scenario

\begin{tabular}{|l|l|}
\hline Vehicle Density & 45 \\
\hline Vehicle Velocity & $15-90 \mathrm{~km} / \mathrm{hr}$ \\
\hline Simulation Duration & 500 seconds \\
\hline Clustering Protocols & StabTrust, DHC, CACONET, and TACA \\
\hline MAC & $802.11 \mathrm{p}$ \\
\hline Propagation Model & Two-Ray Ground \\
\hline Area & $7050 \mathrm{x} 7050$ \\
\hline Mobility Model & Manhattan grid mobility model \\
\hline Traffic Pattern & Constant Bit Ratio (CBR) \\
\hline Communication Pairs & 6 \\
\hline Number of RSUs & 5 \\
\hline
\end{tabular}

Figure 8 shows the performance of average throughput for each clustering protocol with increased vehicle speed. The impact of mobility is indicated by the results of throughput. Higher velocity leads to lower throughput performances using each clustering protocol. The TACA achieved enhanced throughput performance compared to existing clustering protocols because of optimization-based reliable $\mathrm{CH}$ selection and a simplified approach for route discovery compared to DHC, CACONET, and StabTrust protocols. Similarly, figure 9 shows the PDR performance ant it is also affected by the increased mobility speed of vehicles. The TACA protocol able to reduce the data loss compared to other protocols. The existing protocols have failed to consider the congestion and packet delivery ability related parameters along with mobility for optimal $\mathrm{CH}$ selection and route formation, and hence, leads to minimum PDR compared to TACA protocol.

As discussed above concerning communication delay and control overhead, frequent route establishment and clustering in VANETs affect these performances. This frequency of route formation and clustering increases as the mobility of vehicles increases. The results of communication delay (figure 10) and control overhead (figure 11) are showing that how the higher mobility leads to the worst communication delay and overhead in VANETs. The communication delay and control over- 
head performances are reduced using the TACA protocol compared to the existing clustering protocol. These results also justified that for any kind of VANET networks, the performances of space and time complexity is lower using the TACA method as it believes in mobility and congestion aware clustering and data transmissions compared to existing protocols.

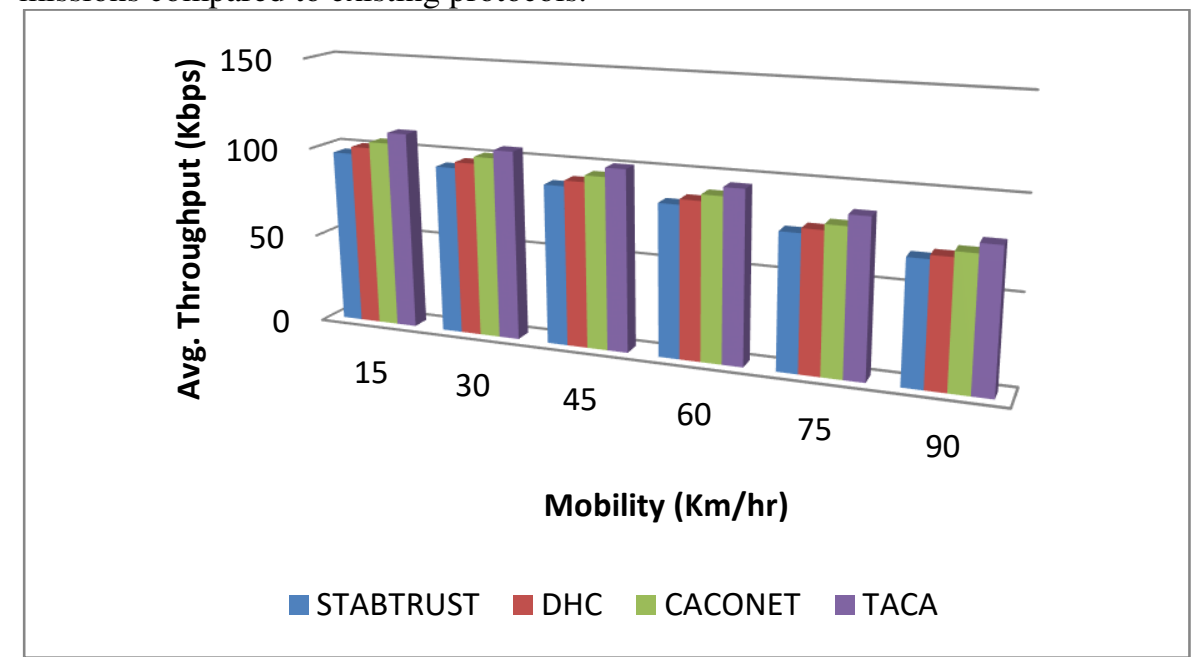

Figure 8. Average throughput analysis for mobility variations

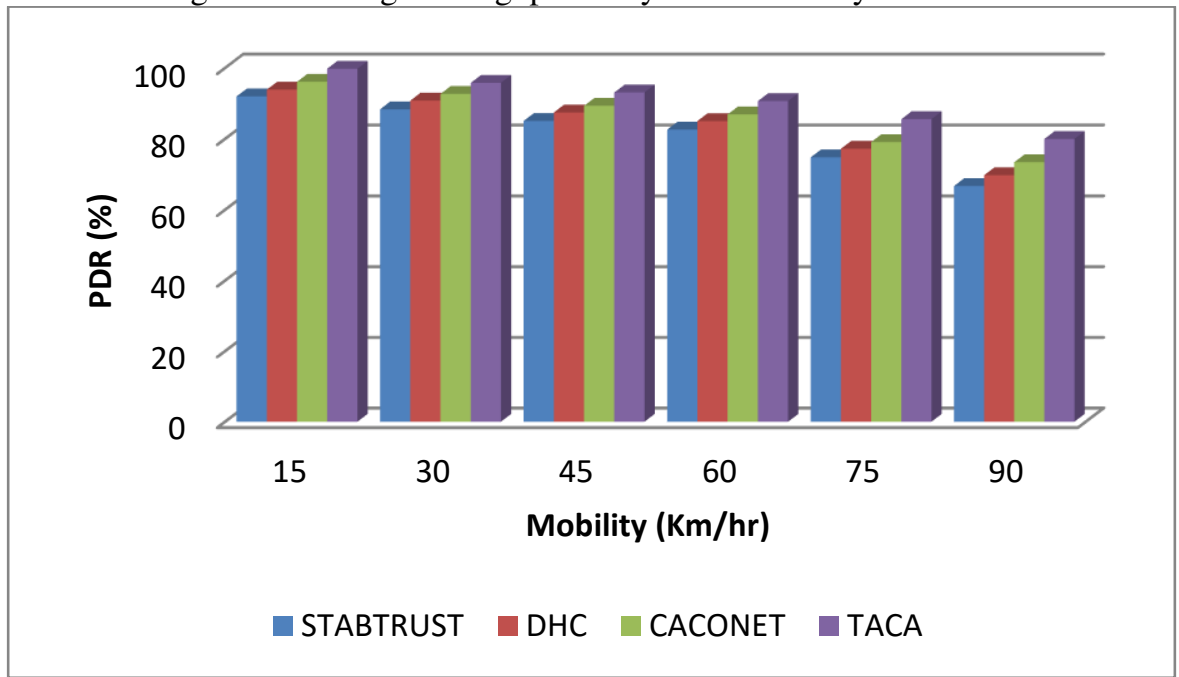

Figure 9. PDR analysis for mobility variations 


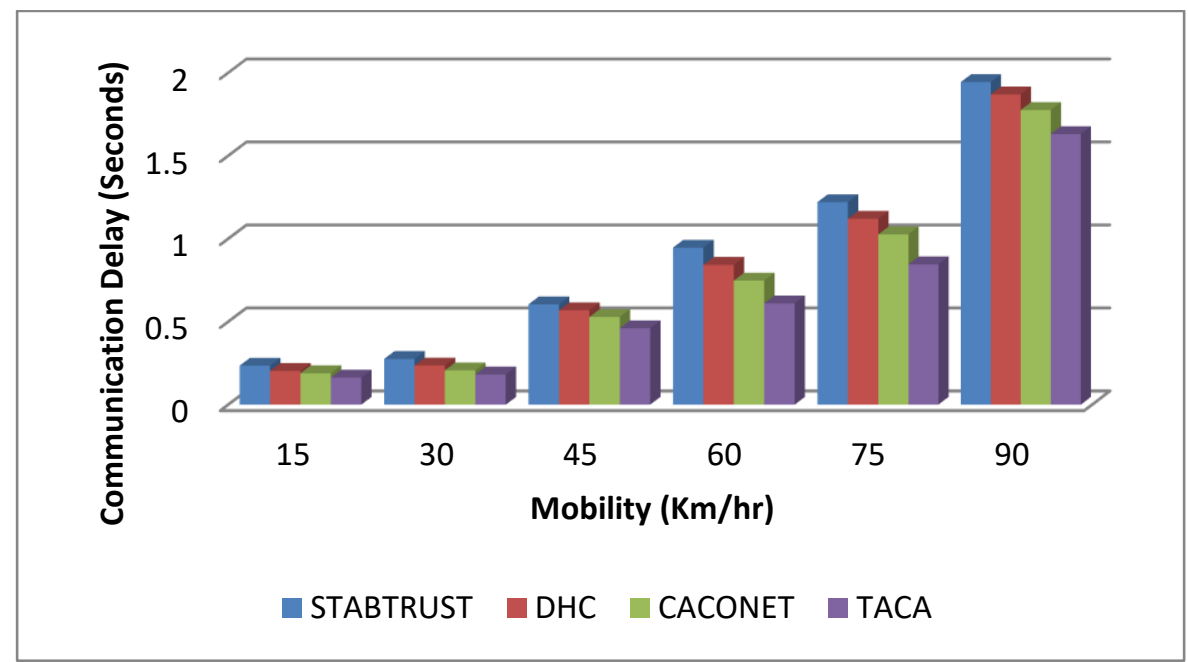

Figure 10. Communication delay analysis for mobility variations

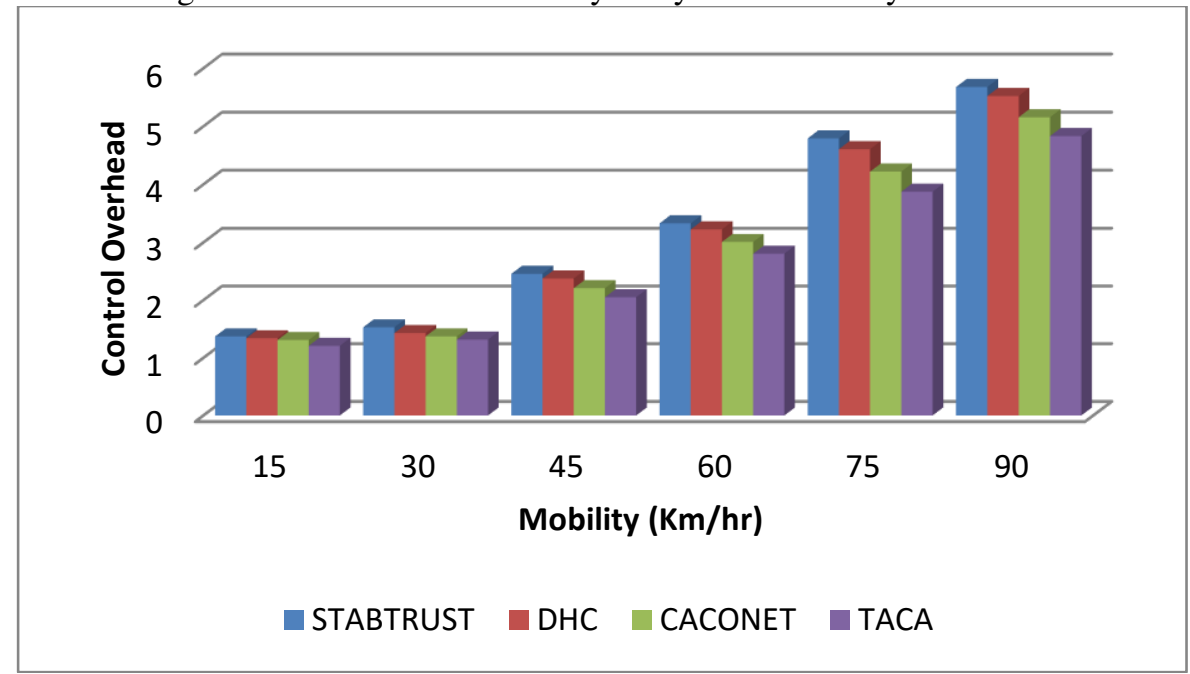

Figure 11. Control overhead analysis for mobility variations

\section{$5 \quad$ Conclusion and Future Work}

This paper proposed the novel trust-based mechanism for VANET clustering called TACA. The main contributions of the TACA protocol are (1) ACO with trust-based fitness computation for reliable and stable $\mathrm{CH}$ selection, (2) Computation of integrated fitness value of each vehicle using congestion, connectivity, and mobility trust parameters, and, (3) lightweight mechanism for reliable relay selection for data forwarding. The ACO optimization technique is used for stable clustering with min- 
imum control overhead and higher QoS performance. We designed network scenarios of density and mobility variations to claim the efficiency of the TACA protocol. The performance of the TACA protocol has compared with DHC, CACONET, and StabTrust clustering protocols in terms of average throughput, PDR, communication delay, and control overhead. Throughput and PDR performance of TACA protocol increased by $9 \%$ and $10.1 \%$ respectively compared to existing protocols. Communication delay and control overhead reduced by $14.5 \%$ and $12.3 \%$ respectively. For future work, we suggest (1) investigate the TACA protocol by introducing the different types of attacks, (2) design route formation algorithms using the ACO, and (3) investigate the other optimization algorithms in place of ACO.

\section{Declarations:}

Funding: No Funding.

Conflict of Interest: All authors declares that they has no conflict of interest.

Availability of data and material: Article does not have any associated dataset.

Code availability: It will be available based on reasonable request.

Ethical approval: This article does not contain any studies with human participants performed by any of the authors.

\section{References}

1. Bachir, A., \& Benslimane, A. (n.d.). A multicast protocol in ad hoc networks inter-vehicle geocast. The 57th IEEE Semiannual Vehicular Technology Conference, 2003. VTC 2003-Spring. doi:10.1109/vetecs.2003.1208832.

2. Benslimane, A. (2004). Optimized Dissemination of Alarm Messages in Vehicular Ad-Hoc Networks (VANET). High Speed Networks and Multimedia Communications, 655-666. doi:10.1007/978-3-540-25969-5_59.

3. Korkmaz, G., Ekici, E., Özgüner, F., \& Özgüner, Ü. (2004). Urban multihop broadcast protocol for inter-vehicle communication systems. Proceedings of the First ACM Workshop on Vehicular Ad Hoc Networks VANET '04. doi:10.1145/1023875.1023887.

4. Joshi, Harshvardhan \& Sichitiu, Mihail \& Kihl, Maria. (2007). Distributed Robust Geocast Multicast Routing for Inter-Vehicle Communication. Proceedings of WEIRD Workshop on WiMax, Wireless and Mobility. 
5. Kihl, M., Sichitiu, M., Ekeroth, T., \& Rozenberg, M. (n.d.). Reliable Geographical Multicast Routing in Vehicular Ad-Hoc Networks. Wired/Wireless Internet Communications, 315-325. doi:10.1007/978-3540-72697-5_27.

6. Mahajan, H.B., Badarla, A. \& Junnarkar, A.A. (2020). CL-IoT: cross-layer Internet of Things protocol for intelligent manufacturing of smart farming. J Ambient Intell Human Comput. https://doi.org/10.1007/s12652-02002502-0.

7. Mahajan, H.B., \& Badarla, A. (2019). Experimental Analysis of Recent Clustering Algorithms for Wireless Sensor Network: Application of IoT based Smart Precision Farming. Jour of Adv Research in Dynamical \& Control Systems, Vol. 11, No. 9. 10.5373/JARDCS/V11I9/20193162.

8. Rawashdeh, Z. Y., \& Mahmud, S. M. (2012). A novel algorithm to form stable clusters in vehicular ad hoc networks on highways. EURASIP Journal on Wireless Communications and Networking, 2012(1). doi:10.1186/1687-1499-2012-15.

9. Fan, P. (2007). Improving Broadcasting Performance by Clustering with Stability for Inter-Vehicle Communication. 2007 IEEE 65th Vehicular Technology Conference - VTC2007-Spring. doi:10.1109/vetecs.2007.513.

10. Cooper, C., Franklin, D., Ros, M., Safaei, F., \& Abolhasan, M. (2017). A Comparative Survey of VANET Clustering Techniques. IEEE Communications Surveys \& Tutorials, 19(1), 657-681. doi:10.1109/comst.2016.2611524.

11. Ren, M., Zhang, J., Khoukhi, L. et al. A review of clustering algorithms in VANETs. Ann. Telecommun. (2021). https://doi.org/10.1007/s12243-02000831-X.

12. Pal, R., Prakash, A., Tripathi, R., \& Singh, D. (2018). Analytical model for clustered vehicular ad hoc network analysis. ICT Express. doi:10.1016/j.icte.2018.01.001.

13. Senouci, O., Harous, S., \& Aliouat, Z. (2020). Survey on vehicular ad hoc networks clustering algorithms: Overview, taxonomy, challenges, and open research issues. International Journal of Communication Systems, e4402. doi:10.1002/dac.4402.

14. Soleymani, S.A., Abdullah, A.H., Hassan, W.H. et al. Trust management in vehicular ad hoc network: a systematic review. J Wireless Com Network 2015, 146 (2015). https://doi.org/10.1186/s13638-015-0353-y.

15. Patel, N. J., \& Jhaveri, R. H. (2015). Trust Based Approaches for Secure Routing in VANET: A Survey. Procedia Computer Science, 45, 592-601. doi:10.1016/j.procs.2015.03.112.

16. Wang, Zhigang \& Liu, Lichuan \& Zhou, Mengchu \& Ansari, Nirwan. (2008). A Position-Based Clustering Technique for Ad Hoc Intervehicle Communication. Systems, Man, and Cybernetics, Part C: Applications and Reviews, IEEE Transactions on. 38. $201 \quad$ - 208. 10.1109/TSMCC.2007.913917.

17. Zhang, Z., Boukerche, A., \& Pazzi, R. (2011). A novel multi-hop clustering scheme for vehicular ad-hoc networks. Proceedings of the 9th ACM 
International Symposium on Mobility Management and Wireless Access MobiWac '11. doi:10.1145/2069131.2069135.

18. Dror, E., Avin, C., \& Lotker, Z. (2011). Fast randomized algorithm for hierarchical clustering in Vehicular Ad-Hoc Networks. 2011 The 10th IFIP Annual Mediterranean Ad Hoc Networking Workshop. doi:10.1109/medhoc-net.2011.5970488.

19. Ahizoune, A., \& Hafid, A. (2012). A new stability based clustering algorithm (SBCA) for VANETs. 37th Annual IEEE Conference on Local Computer Networks -- Workshops. doi:10.1109/lcnw.2012.6424072.

20. Lai, Y.-C., Lin, P., Liao, W., \& Chen, C.-M. (2011). A Region-Based Clustering Mechanism for Channel Access in Vehicular Ad Hoc Networks. IEEE Journal on Selected Areas in Communications, 29(1), 83-93. doi:10.1109/jsac.2011.110109.

21. Kakkasageri, M.S. \& Manvi, Sunil. (2012). Multiagent driven dynamic clustering of vehicles in VANETs. Journal of Network and Computer Applications. 35. 1771-1780. 10.1016/j.jnca.2012.07.002.

22. Lo, Shou-Chih \& Lin, Yi-Jen \& Gao, Jhih-Siao. (2013). A Multi-Head Clustering Algorithm in Vehicular Ad Hoc Networks. International Journal of Computer Theory and Engineering. 5. 242-247. 10.7763/IJCTE.2013.V5.686.

23. Aadil, F., Bajwa, K. B., Khan, S., Chaudary, N. M., \& Akram, A. (2016). CACONET: Ant Colony Optimization (ACO) Based Clustering Algorithm for VANET. PLOS ONE, 11(5), e0154080. doi:10.1371/journal.pone.0154080.

24. Daknou, E., Thaalbi, M., \& Tabbane, N. (2015). A Fast Clustering Algorithm for VANETs. Proceedings of the 13th International Conference on Advances in Mobile Computing and Multimedia - MoMM 2015. doi:10.1145/2837126.2837147.

25. Dhugga, P. K., Sharma, M., \& Sharma, A. (2015). An Algorithm for Static Geographical clustering in VANET. 2015 IEEE 3rd International Conference on MOOCs, Innovation and Technology in Education (MITE). doi:10.1109/mite.2015.7375357.

26. ÇALHAN, A. (2015). A fuzzy logic based clustering strategy for improving vehicular ad-hoc network performance. Sadhana, 40(2), 351-367. doi:10.1007/s12046-014-0315-9.

27. Malathi, A. \& Sreenath, N.. (2017). An efficient clustering algorithm for vanet. International Journal of Applied Engineering Research. 12. 20002005.

28. Ren, M., Khoukhi, L., Labiod, H., Zhang, J., \& Vèque, V. (2017). A mobility-based scheme for dynamic clustering in vehicular ad-hoc networks (VANETs). Vehicular Communications, 9, 233-241. doi:10.1016/j.vehcom.2016.12.003.

29. Mohammed Nasr, M., Abdelgader, A., Wang, Z.-G., \& Shen, L.-F. (2016). VANET Clustering Based Routing Protocol Suitable for Deserts. Sensors, 16(4), 478. doi:10.3390/s16040478. 
30. Abuashour, A., \& Kadoch, M. (2017). Performance Improvement of Cluster-Based Routing Protocol in VANET. IEEE Access, 5, 15354-15371. doi:10.1109/access.2017.2733380.

31. Regin, R., Menakadevi, T. Dynamic Clustering Mechanism to Avoid Congestion Control in Vehicular Ad Hoc Networks Based on Node Density. Wireless Pers Commun 107, 1911-1931 (2019). https://doi.org/10.1007/s11277-019-06366-2.

32. Alsuhli, G. H., Khattab, A., \& Fahmy, Y. A. (2019). Double-Head Clustering for Resilient VANETs. Wireless Communications and Mobile Computing, 2019, 1-17. doi:10.1155/2019/2917238.

33. Awan, K. A., Din, I. U., Almogren, A., Guizani, M., \& Khan, S. (2020). StabTrust - A Stable and Centralized Trust-based Clustering Mechanism for IoT enabled Vehicular Ad-hoc Networks. IEEE Access, 1-1. doi:10.1109/access.2020.2968948.

34. Ahsan, W., Khan, M. F., Aadil, F., Maqsood, M., Ashraf, S., Nam, Y., \& Rho, S. (2020). Optimized Node Clustering in VANETs by Using Meta$\begin{array}{llll}\text { Heuristic Algorithms. } & \text { Electronics, }\end{array}$ doi:10.3390/electronics9030394.

35. Senouci, O., Aliouat, Z., \& Harous, S. (2018). A review of routing protocols in internet of vehicles and their challenges. Sensor Review. doi:10.1108/sr-08-2017-0168.

36. Allani, S., Yeferny, T., \& Chbeir, R. (2018). A scalable data dissemination protocol based on vehicles trajectories analysis. Ad Hoc Networks, 71, 3144. doi:10.1016/j.adhoc.2017.12.003.

37. Manzoor, A., Shah, M. A., Khattak, H. A., Din, I. U., \& Khan, M. K. (2019). Multi-tier authentication schemes for fog computing: Architecture, security perspective, and challenges. International Journal of Communication Systems, e4033. doi:10.1002/dac.4033.

38. Khattak, Hasan Ali \& Ameer, Zoobia \& Ud Din, Ikram \& Khan, Muhammad. (2019). Cross-layer design and optimization techniques in wireless multimedia sensor networks for smart cities. Computer Science and Information Systems. 16. 4-4. 10.2298/CSIS181115004K. 